\title{
SEQUENCES WITH MINIMAL TIME-FREQUENCY SPREADS
}

\author{
Reza Parhizkar, Yann Barbotin and Martin Vetterli \\ School of Computer and Communication Sciences \\ Ecole Polytechnique Fédérale de Lausanne (EPFL), CH-1015 Lausanne, Switzerland \\ \{reza.parhizkar, yann.barbotin, martin.vetterli\}@epfl.ch
}

\begin{abstract}
For a given time or frequency spread, one can always find continuoustime signals, which achieve the Heisenberg uncertainty principle bound. This is known, however, not to be the case for discrete-time sequences; only widely spread sequences asymptotically achieve this bound. We provide a constructive method for designing sequences that are maximally compact in time for a given frequency spread. By formulating the problem as a semidefinite program, we show that maximally compact sequences do not achieve the classic Heisenberg bound. We further provide analytic lower bounds on the time-frequency spread of such signals.
\end{abstract}

Index Terms - Heisenberg Uncertainty Principle, Compact Sequences, Harmonic Analysis, Filter Design, Semidefinite Programming

\section{INTRODUCTION}

Consider the common problem of filter design; we are asked to design a filter that is compact both in time and frequency domains. To this end, the notion of compactness and spread must be defined correctly. These notions are very well defined and established for continuous-time signals $[1,2]$. For a continuous-time signal, we can define the time and frequency characteristics of a signal as in Table 1.

The Heisenberg uncertainty principle [1] states that continuoustime signals cannot be arbitrarily compact in both domains. Specifically,

For any $x(t) \in L^{2}(\mathbb{R})$

$$
\eta_{c}=\Delta_{t}^{2} \Delta_{\omega_{c}}^{2} \geq \frac{1}{4}
$$

where the lower bound is achieved for Gaussian signals of the form $x(t)=\gamma e^{-\alpha t^{2}}, \alpha>0$. The subscript $c$ stands for continuous-time definitions. We call $\eta_{c}$ the time-frequency spread of $x$.

One can easily see the connection of these definitions with the mean and variance of a probability distribution function $|x(t)|^{2} /\|x\|^{2}$ (under some smoothness conditions for $x(t)$ ). Although the continuous Heisenberg uncertainty principle is widely used in theory, in practice we mostly work with discrete-time signals (e.g. filters and wavelets). Thus, equivalent definitions for discrete-time sequences are needed in signal processing.

This work was supported by ERC Advanced Grant-Support for Frontier Research-SPARSAM, Nr: 247006.

\subsection{Definitions}

An obvious and intuitive extension of the definitions in Table 1 for discrete-time signals is presented in Table 2, where

$$
X\left(e^{j \omega}\right)=\sum_{n \in \mathbb{Z}} x_{n} e^{-j \omega n} \quad \omega \in \mathbb{R},
$$

is the discrete-time Fourier transform (DTFT) of $x_{n}$.

Using the definitions in Table 2, we can also state the Heisenberg uncertainty principle for discrete-time signals as [2]

For a discrete-time sequence $x_{n} \in \ell^{2}(\mathbb{Z})$ with $X\left(e^{j \pi}\right)=0$,

$$
\eta_{\ell}=\Delta_{n}^{2} \Delta_{\omega_{\ell}}^{2}>\frac{1}{4},
$$

where the subscript $\ell$ stands for linear in reference to the definition of the frequency spread. Note the extra assumption on the Fourier transform of the signal for (3) to hold. This assumption is necessary for the result to hold.

Example: Take $x_{n}=\delta_{n}+4 \delta_{n-1}+2 \delta_{n-2}$. The Fourier transform of $x_{n}$ is shown in Figure 1. Observe that $\left|X\left(e^{j \pi}\right)\right|=$ $0.09 \neq 0$, which violates the condition $X\left(e^{j \pi}\right)=0$. The linear time-frequency spread of this signal according to Table 2 is $\eta_{\ell}=$ $0.239<1 / 4$.

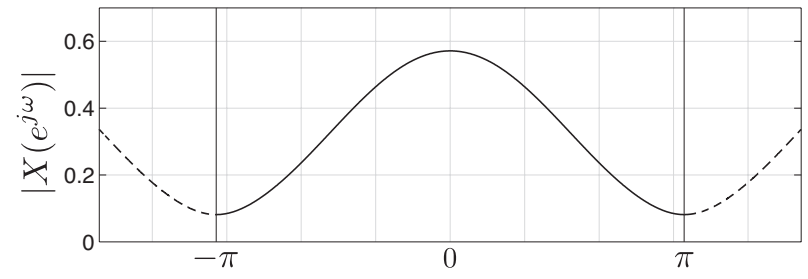

Fig. 1. A signal that violates $X\left(e^{j \pi}\right)=0$ and does not satisfy the linear Heisenberg uncertainty principle $\left(\eta_{\ell}=0.239<\frac{1}{4}\right)$.

In addition to the restriction on the Heisenberg uncertainty principle, the definitions in Table 2 do not capture the periodic nature of $X\left(e^{j \omega}\right)$ for the frequency center and spread. To overcome these shortcomings, we can adopt definitions for circular moments widely used in quantum mechanics [3] and directional statistics [4].

For a sequence $x_{n}, n \in \mathbb{Z}$, with a $2 \pi$ periodic DTFT, $X\left(e^{j \omega}\right)$ as in (2), the first trigonometric moment is defined as [5,6]

$$
\tau(x)=\frac{1}{2 \pi\|x\|^{2}} \int_{-\pi}^{\pi} e^{j \omega}\left|X\left(e^{j \omega}\right)\right|^{2} d \omega .
$$

The first trigonometric moment is originally defined for probability distributions on a circle. With proper normalization, this definition applies also to periodic functions. 


\begin{tabular}{lll}
\hline domain & center & spread \\
\hline time & $\mu_{t}=\frac{1}{\|x\|^{2}} \int_{t \in \mathbb{R}} t|x(t)|^{2} d t$ & $\Delta_{t}^{2}=\frac{1}{\|x\|^{2}} \int_{t \in \mathbb{R}}\left(t-\mu_{t}\right)^{2}|x(t)|^{2} d t$ \\
frequency & $\mu_{\omega_{c}}=\frac{1}{2 \pi\|x\|^{2}} \int_{\omega \in \mathbb{R}} \omega|X(\omega)|^{2} d \omega$ & $\Delta_{\omega_{c}}^{2}=\frac{1}{2 \pi\|x\|^{2}} \int_{\omega \in \mathbb{R}}\left(\omega-\mu_{\omega}\right)^{2}|X(\omega)|^{2} d \omega$ \\
\hline
\end{tabular}

Table 1. Time and frequency centers and spreads for a continuous time signal $x(t)$.

\begin{tabular}{lll}
\hline domain & center & spread \\
\hline time & $\mu_{n}=\frac{1}{\|x\|^{2}} \sum_{n \in \mathbb{Z}} n\left|x_{n}\right|^{2}$ & $\Delta_{n}^{2}=\frac{1}{\|x\|^{2}} \sum_{n \in \mathbb{Z}}\left(n-\mu_{n}\right)^{2}\left|x_{n}\right|^{2}$ \\
frequency & $\mu_{\omega_{\ell}}=\frac{1}{2 \pi\|x\|^{2}} \int_{-\pi}^{\pi} \omega\left|X\left(e^{j \omega}\right)\right|^{2} d \omega$ & $\Delta_{\omega_{\ell}}^{2}=\frac{1}{2 \pi\|x\|^{2}} \int_{-\pi}^{\pi}\left(\omega-\mu_{\omega}\right)^{2}\left|X\left(e^{j \omega}\right)\right|^{2} d \omega$ \\
\hline
\end{tabular}

Table 2. Time and frequency centers and spreads for a discrete time signal $x_{n}$ as extensions of Table 1 [2].

Using (4), the periodic frequency spread is defined as [3]

$$
\Delta_{\omega_{p}}^{2}=\frac{1-|\tau(x)|^{2}}{|\tau(x)|^{2}}=\left|\frac{\|x\|^{2}}{\sum_{n \in \mathbb{Z}} x_{n} x_{n+1}^{*}}\right|^{2}-1 .
$$

The definition of $\Delta_{n}^{2}$ remains unchanged as in Table 2. These definitions are summarized in Table 3.

\subsection{Contributions}

In this paper, using the definitions in Table 3, we revisit the Heisenberg uncertainty principle for discrete-time signals.

We address the fundamental yet unanswered question : If someone asks us to design a discrete filter with a certain frequency spread ( $\Delta_{\omega_{p}}^{2}$ fixed), can we return the sequence with minimal time spread $\Delta_{n}^{2}$ ? In other words, the problem is to find the solution to

$$
\begin{aligned}
\Delta_{n, \mathrm{opt}}^{2}=\underset{x_{n}}{\operatorname{minimize}} \quad \Delta_{n}^{2} \\
\text { subject to } \Delta_{\omega_{p}}^{2}=\text { fixed } .
\end{aligned}
$$

We call the solution of (6) a maximally compact sequence. Framing the design of maximally compact sequences as an optimization problem, we show that in contrary to the continuous case, it is not possible to reach a constant time-frequency lower bound for arbitrary time or frequency spreads. We further develop a simple optimization framework to find maximally compact sequences in the time domain for a given frequency spread. In other words, we provide in a constructive and numerical way, a sharp uncertainty principle for sequences.

\subsection{Related Work}

The classic uncertainty principle [1] assumes continuoustime/continuous-frequency signals. Several works in the signal processing community also address the discrete-time/discretefrequency case [7-9]. Our work bridges between these two cases by considering the discrete-time/continuous-frequency regime.

Note that not all studies about uncertainty principle concern the notion of spread. For example, the authors in [8] propose the uncertainty bound on the information content of signals (entropy) and [9] provides a bound on the non-zero coefficients of discrete-time sequences and their discrete Fourier transforms.
The discrete-time/continuous-frequency scenario has been recently encountered in many practical applications in signal processing. Examples include uncertainty principle on graphs [10] and on spheres [11]. Initial studies on the periodic frequency spread were undertaken by [3] and [12]. The most comprehensive work on the uncertainty relations for discrete sequences is found in [6]. The authors show that $1 / 4$ is a lower-bound on the time-frequency spread, which can only be achieved asymptotically as the sequence spreads in time.

This problem has some analogy with the design of Slepian's Discrete Prolate Spheroidal Sequences (DPSS's) [13]. However, in contrary to these sequences, we do not impose any constraints on the bandwidth of the sequence in the frequency domain; instead, we use the notion of variance as a measure of concentration in time and frequency ${ }^{1}$.

\subsection{Organization}

The organization of the paper is as follows. In Section 2, we visit the Heisenberg uncertainty principle for discrete sequences. In Section 3 , we identify some properties of maximally compact sequences. Then in Section 4, we show how to use a semidefinite relaxation to solve the problem (6). In Section 5, we provide an analytic lower bound on the solution of the optimization problem and finally in Section 6, we present some experimental results for constructing maximally compact sequences.

\section{HEISENBERG UNCERTAINTY PRINCIPLE FOR PERIODIC SPECTRUMS}

Although the definition of the frequency spread in Table 3 is less intuitive, Breitenberger proves in [3] that for a concentrated periodic distribution, $\Delta_{\omega_{p}}^{2}$ in (5) converges to the linear variance $\Delta_{\omega_{\ell}}^{2}$. Also viewing frequency as a phase derivative, Torresani gives a nice justification of this formulation in [14].

The definitions in Table 3 take into account the periodic nature of the Fourier transform of the signal and in this sense are optimal for characterizing the frequency spread of discrete-time sequences.

Using the definitions in Table 3, the periodic Heisenberg uncertainty principle reads as follows [3]:

\footnotetext{
${ }^{1}$ The authors thank the anonymous reviewer for suggesting the connection with the DPSS's.
} 


\begin{tabular}{lll}
\hline domain & center & spread \\
\hline time & $\mu_{n}=\frac{1}{\|x\|^{2}} \sum_{n \in \mathbb{Z}} n\left|x_{n}\right|^{2}$ & $\Delta_{n}^{2}=\frac{1}{\|x\|^{2}} \sum_{n \in \mathbb{Z}}\left(n-\mu_{n}\right)^{2}\left|x_{n}\right|^{2}$ \\
frequency & $\mu_{\omega_{p}}=\arg \left[\int_{-\pi}^{\pi} e^{j \omega}\left|X\left(e^{j \omega}\right)\right|^{2} d \omega\right]$ & $\Delta_{\omega_{p}}^{2}=\left|\frac{\|x\|^{2}}{\sum_{n \in \mathbb{Z}} x_{n} x_{n+1}^{*}}\right|^{2}-1$ \\
\hline
\end{tabular}

Table 3. Time and frequency centers and spreads for a discrete time signal $x_{n}$ using circular moments.

For a discrete-time sequence $x_{n} \in \ell^{2}(\mathbb{Z})$, with $\left\|x_{n}\right\|_{0}>1$,

$$
\eta_{p}=\Delta_{n}^{2} \Delta_{\omega_{p}}^{2}>\frac{1}{4}
$$

This principle is used mostly for periodic signals with discrete Fourier series coefficients. Here, we use the duality of the Fourier transform to define them for discrete-time sequences and their DTFTs. It is proved in [5] that the lower bound cannot be achieved in general. The authors propose a framework for finding functions which reach asymptotically the bound. However, this asymptotic regime is reached for very large time spreads and frequency spreads vanishing to 0 , which seriously reduces its range of application ${ }^{2}$.

\section{PROPERTIES OF MAXIMALLY COMPACT SEQUENCES}

So far, we have considered arbitrary complex sequences and their DTFT spectrum. In the following, we establish two lemmas that make the search for maximally compact sequences more tractable.

Lemma 1. Maximally compact sequences are generalized linearphase sequences derived from real-valued, positive maximally compact sequences; i.e. $x_{n}$ is a maximally compact sequence only if

$$
\exists \varphi, \psi \in\left[0,2 \pi\left[\text { such that } x_{n}=\left|x_{n}\right| e^{-j(\varphi n+\psi)},\right.\right.
$$

where $|x|$ is a maximally compact sequence.

Proof. For the proof, we refer the reader to the supplementary material provided in [15].

Consider also the shift operator

$$
x_{n+\nu} \stackrel{\text { DTFT }}{\longleftrightarrow} e^{j \omega \nu} X\left(e^{j \omega}\right), \nu \in \mathbb{R},
$$

whose principal effect is to shift the time centre of a sequence

$$
\mu_{n}\left(x_{n+\nu}\right)=\mu_{n}(x)+\nu .
$$

Notice that $\nu$ might not be integer, in which case $x_{n-\nu}$ is a shorthand for sinc resampling on a grid shifted by $\nu$ in the time-domain.

Lemma 2. If $x$ is a maximally compact sequence, then $x_{n-\mu_{n}(x)}$ is also maximally compact.

Proof. The proof is provided in [15].

Lemmas 1 and 2 greatly reduce the complexity of the problem, and from here on we only consider-without loss of generalityreal, positive sequences $x$, with $\mu_{n}(x)=0$ and $\|x\|^{2}=1$. It also shows that the uncertainty principle for sequences will retain a probabilistic interpretation similar to the Heisenberg uncertainty for functions.

\footnotetext{
${ }^{2}$ In this asymptotic regime, the sequence and its spectrum share many properties with their continuous-time counterparts. So, the similarity with the continuous-time Heisenberg uncertainty principle is not surprising.
}

\section{REFORMULATION AS A QCQP}

If we are given a fixed frequency spread $\Delta_{\omega_{p}}^{2}=\sigma^{2}$, by using Lemmas 1 and 2, we can write problem (6) for finding maximally compact sequences in the time domain as

$$
\begin{aligned}
\Delta_{n, \mathrm{opt}}^{2}=\underset{x_{n}}{\operatorname{minimize}} & \sum_{n \in \mathbb{Z}} n^{2} x_{n}^{2} \\
\text { subject to } & \sum_{n \in \mathbb{Z}} x_{n} x_{n+1}=\frac{1}{\sqrt{1+\sigma^{2}}}, \\
& \sum_{n \in \mathbb{Z}} x_{n}^{2}=1 .
\end{aligned}
$$

Note that the constraint comes from the definition of $\Delta_{\omega_{p}}^{2}$ in Table 3. We can rewrite (11) in a matrix form as a quadratically constrained quadratic program (QCQP) [16]

$$
\begin{array}{cl}
\underset{\boldsymbol{x}}{\operatorname{minimize}} & \boldsymbol{x}^{T} \boldsymbol{A} \boldsymbol{x} \\
\text { subject to } & \boldsymbol{x}^{T} \boldsymbol{B} \boldsymbol{x}=\alpha, \\
& \boldsymbol{x}^{T} \boldsymbol{x}=1,
\end{array}
$$

where

$$
\boldsymbol{A}=\left[\begin{array}{lllllll}
\ddots & & & & & & \\
& 2^{2} & & & & 0 & \\
& & 1^{2} & & & & \\
& & & 0 & & & \\
& & & 1^{2} & & \\
& 0 & & & 2^{2} & \\
& & & & &
\end{array}\right], \quad \boldsymbol{B}=\left[\begin{array}{cccccc}
\ddots & & & & 0 \\
\frac{1}{2} & 0 & \frac{1}{2} & & \\
& \frac{1}{2} & 0 & \frac{1}{2} & \\
& & \frac{1}{2} & 0 & \frac{1}{2} \\
0 & & & & \ddots
\end{array}\right],
$$

and $\alpha=1 / \sqrt{1+\sigma^{2}}$. This problem can be further reformulated as follows:

$$
\begin{array}{cl}
\underset{\boldsymbol{x}}{\operatorname{minimize}} & \operatorname{tr}\left(\boldsymbol{A} \boldsymbol{x} \boldsymbol{x}^{T}\right) \\
\text { subject to } & \operatorname{tr}\left(\boldsymbol{B} \boldsymbol{x} \boldsymbol{x}^{T}\right)=\alpha \\
& \operatorname{tr}\left(\boldsymbol{x} \boldsymbol{x}^{T}\right)=1 .
\end{array}
$$

Replacing $\boldsymbol{x} \boldsymbol{x}^{T}$ by $\boldsymbol{X}$, we can write equivalently

$$
\begin{array}{cl}
\underset{\boldsymbol{X}}{\operatorname{minimize}} & \operatorname{tr}(\boldsymbol{A} \boldsymbol{X}) \\
\text { subject to } & \operatorname{tr}(\boldsymbol{B} \boldsymbol{X})=\alpha \\
& \operatorname{tr}(\boldsymbol{X})=1 \\
& \boldsymbol{X} \succeq 0, \operatorname{rank}(\boldsymbol{X})=1 .
\end{array}
$$

We can relax the above formulation to reach the semidefinite program

$$
\begin{array}{ll}
\underset{\boldsymbol{X}}{\operatorname{minimize}} & \operatorname{tr}(\boldsymbol{A} \boldsymbol{X}) \\
\text { subject to } & \operatorname{tr}(\boldsymbol{B} \boldsymbol{X})=\alpha \\
& \operatorname{tr}(\boldsymbol{X})=1, \quad \boldsymbol{X} \succeq 0 .
\end{array}
$$


Lemma 3. The semidefinite relaxation (SDR) in (13) is tight.

Proof. Shapiro and then Barnivok and Pataki [17-20] show that if the SDP in (12) is feasible, then

$$
\operatorname{rank}\left(\boldsymbol{X}^{\mathrm{opt}}\right) \leq\lfloor(\sqrt{8 m+1}-1) / 2\rfloor,
$$

where $m$ is the number of constraints of the SDP and $\boldsymbol{X}^{\mathrm{opt}}$ is its optimal solution. For our semidefinite program in (13), $m=2$. Thus, (14) implies that the solution has rank 1. Using this fact, one can see that the semidefinite relaxation is in fact tight. Note that from the nature of the problem, (12) is clearly feasible; We can always find a periodic signal in the Fourier domain with a unit norm and a desired frequency spread, although not having an optimal time spread.

The SDP in (13) can be solved to any precision by using the existing approaches in the optimization literature; for example using the CVX software package [21].

\section{ANALYTIC LOWER BOUND}

Note that by solving (13), one can constructively produce maximally compact sequences upto machine precision. As shown in Section 6, these sequences do not reach the Heisenberg bound. In this section we provide an analytic lower bound on the solution of (13).

The dual of SDR (13) is [16]

$$
\begin{array}{ll}
\underset{\lambda_{1}, \lambda_{2}}{\operatorname{maximize}} & \alpha \lambda_{1}+\lambda_{2} \\
\text { subject to } & \boldsymbol{A}-\lambda_{1} \boldsymbol{B}-\lambda_{2} \boldsymbol{I} \succeq 0
\end{array}
$$

Lemma 4. For the primal problem (13) and the dual (15), strong duality holds.

Proof. We refer the reader to [15] for the proof.

Thus, for finding the time-frequency spread of maximally compact sequences, solving the dual problem suffices. In the following theorem we show that the solution to the dual problem (and thus the primal) is bounded from below.

Theorem 1. If $x_{n}$ is maximally compact for a given $\Delta_{\omega_{p}}^{2}=\sigma^{2}$, then

$$
\eta_{p}=\Delta_{n}^{2} \Delta_{\omega_{p}}^{2} \geq \sigma^{2}\left(1-\sqrt{\frac{\sigma^{2}}{1+\sigma^{2}}}\right) .
$$

Proof. The detailed proof is provided in [15].

This is a fundamental result showing that although the Heisenberg uncertainty principle provides a lower bound for the timefrequency spread of the signal, it cannot be achieved for any given frequency (or equivalently time) spread.

\section{EXPERIMENTAL RESULTS}

In order to evaluate the results of this paper, we perform simulations to find the optimally compact sequences for a given frequency spread. We solve the semidefinite program (13) using the cvx package.

Figure 2 shows the Fourier transform of the resulting sequences for different input values of $\Delta_{\omega_{p}}^{2}$. Note that we can find $\boldsymbol{x}^{\text {opt }}$ from $\boldsymbol{X}^{\text {opt }}$ with a simple singular value decomposition, as we have shown that $\boldsymbol{X}^{\mathrm{opt}}$ has rank 1.

Further, Figure 3 shows the time-frequency spread, $\eta_{p}$ of the resulting signals for given values of $\Delta_{\omega_{p}}^{2}$. One can observe that, in

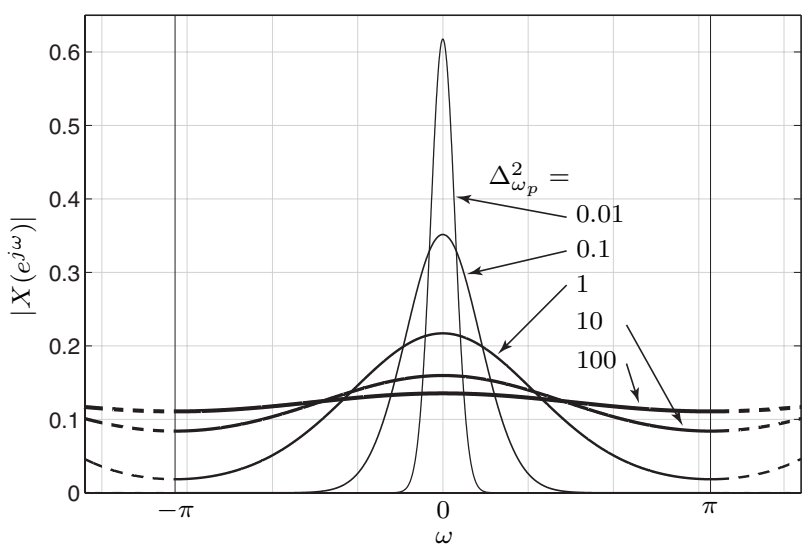

Fig. 2. For a fixed frequency spread $\Delta_{\omega_{p}}^{2}$, the spectrum of the solutions to the SDR problem are at numerical precision similar to wrapped-gaussian kernels.

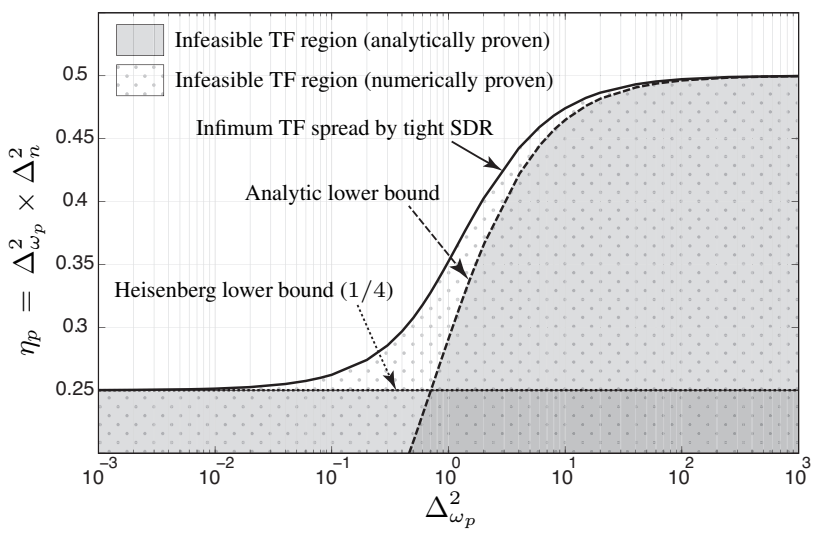

Fig. 3. Achievability of the discrete time-frequency spread $\eta_{p}$ conditionned on the frequency spread. The analytic lower bound becomes tight as the frequency spread increases.

contrary to continuous-time signals, maximally compact sequences do not reach the Heisenberg lower bound. Also, the analytic bound in Theorem 1 converges to $1 / 2$ as $\Delta_{\omega_{p}}^{2}$ grows, and its union with Heisenberg lower bound crudely delineates the achievable timefrequency spread region.

\section{CONCLUSION}

Using the terminology from [3], we introduce a constructive method for designing maximally compact sequences with a given frequency (or equivalently time) spread. We provide an analytical lower bound that shows that the time-frequency spread of narrow in time sequences is bounded from below by $1 / 2$ and not just $1 / 4$.

The maximally compact sequences constructed with the presented method provide a sharp uncertainty principle for sequences.

All the results presented in this article are reproducible. Codes for generating the figures are available at http://rr.epfl.ch. 


\section{References}

[1] W. Heisenberg, "translated: The actual content of quantum theoretical kinematics and mechanics," Physikalishche Z., vol. 43, pp. 172, 1927.

[2] M. Vetterli, J. Kovacevic, and V. K. Goyal, Foundations of Signal Processing, 2012, http://www.fourierandwavelets.org/.

[3] E. Breitenberger, "Uncertainty measures and uncertainty relations for angle observables," Foundations of Physics, vol. 15, no. 3, pp. 353-364, 1985.

[4] K. V. Mardia and P. E. Jupp, Directional Statistics, Wiley, 2009.

[5] J. Prestin and E. Quak, "Optimal functions for a periodic uncertainty principle and multiresolution analysis," Proceedings of the Edinburgh Mathematical Society, vol. 42, no. 2, pp. 225242, 1999.

[6] J. Prestin, E. Quak, H. Rauhut, and K. Selig, "On the connection of uncertainty principles for functions on the circle and on the real line," Journal of Fourier Analysis and Applications, vol. 9, no. 4, pp. 387-409, 2003.

[7] S. Massar and Ph. Spindel, "Uncertainty relation for the discrete Fourier transform," Phys. Rev. Lett., vol. 100, pp. 190401 , May 2008.

[8] T. Przebinda, V. DeBrunner, and M. Ozaydin, "Using a new uncertainty measure to determine optimal bases for signal representations," in IEEE International Conference on Acoustics, Speech and Signal Processing (ICASSP), 1999.

[9] D. L. Donoho and P. B. Stark, "Uncertainty principles and signal recovery," SIAM Journal on Applied Mathematics, vol. 49, no. 3, pp. 906-931, 1989.

[10] A. Agaskar and Y. M. Lu, "Uncertainty principles for signals defined on graphs: Bounds and characterizations," in IEEE International Conference on Acoustics, Speech and Signal Processing (ICASSP), 2012.

[11] Z. Khalid, S. Durrani, P. Sadeghi, and R. A. Kennedy, "Concentration uncertainty principles for signals on the unit sphere," in IEEE International Conference on Acoustics, Speech and Signal Processing (ICASSP), 2012.

[12] R. von Mises, "Über die "Ganzzahligkeit" der Atomgewichte und verwandte Fragen," Physikalishche Z., vol. 19, pp. 490$500,1918$.

[13] D. Slepian, "Prolate spheroidal wave functions, Fourier analysis, and uncertainty. V:The discrete case," vol. 57, no. 5, pp. 1371-1430, 1978.

[14] B. Torresani, "Position-frequency analyis for signals defined on spheres," Signal Processing, vol. 43, no. 3, pp. 341-346, 1995.

[15] R. Parhizkar, Yann Barbotin, and M. Vetterli, "Sequences with minimal time-frequency uncertainty," 2013, submitted to Applied and Computational Harmonic Analysis (ACHA), http://arxiv.org/abs/1302.2082.
[16] S. Boyd and L. Vandenberghe, Convex optimization, Cambridge University Press, 2004.

[17] A. Shapiro, "Rank-reducibility of a symmetric matrix and sampling theory of minimum trace factor analysis," Psychometrika, vol. 47, no. 2, pp. 187-199, 1982.

[18] A.I. Barvinok, "Problems of distance geometry and convex properties of quadratic maps," Discrete \& Computational Geometry, vol. 13, no. 1, pp. 189-202, 1995.

[19] G. Pataki, "On the rank of extreme matrices in semidefinite programs and the multiplicity of optimal eigenvalues," Mathematics of Operations Research, pp. 339-358, 1998.

[20] Z. Luo, W. Ma, A. M. C. So, Y. Ye, and S. Zhang, "Semidefinite relaxation of quadratic optimization problems," IEEE Signal Processing Magazine, vol. 27, no. 3, pp. 20-34, 2010.

[21] M. Grant and S. Boyd, "cvx: Matlab software for disciplined convex programming, version 1.21," 2010. 